\title{
Review of: "Systematic review and meta-analysis of modified facelift incision versus modified Blair incision in parotidectomy"
}

Qiang Zhang

Potential competing interests: The author(s) declared that no potential competing interests exist.

Surgical resection is recommended as the preferred treatment for benign parotid tumours, but traditionally parotidectomy leaves a permanent visible scar postoperatively, which is usually unacceptable to young patients, especially young women. In the recent two decades, owing to the adequate knowledge of benign salivary tumors relating to pathological and biological entities, such as pleomorphic adenoma, many oral surgeons have adopted partial superficial parotidectomy (PSP) or extracapsular parotidectomy (ECP) to manage benign tumours, which are less invasive comparing with traditional approach. Based on these developments, different types of cosmetic incisions have been employed for surgery of benign parotid neoplasms, and satisfactory results have been reported in many literature. These are consistent with our clinical experience. However, no meta-analysis has been performed to evaluate the difference between these techniques in parotidectomy before Lee's report. The outcomes of his meta-analysis showed the patients who underwent parotidectomy with the modified facelift incision (MFI) demonstrated signicantly higher cosmetic satisfaction and lower incidence of postoperative Frey's syndrome than those who underwent $\mathrm{MBI}$, but with a similar rate of postoperative salivary complications, temporary and permanent facial palsy. This work also provided evidence and basis for us to perform different cosmetic incisions in parotidectomy to pursue satisfactory result. In addition, the review showed the incision can be provide adequate surgical field exposure and lesion access for tumor excision without jeopardizing oncologic safety. I think this is MORE important than cosmetic incision. 